\title{
Original
}

\section{Effects of microwave tissue coagulation on postoperative complications in patients who underwent liver resection}

\author{
Shoji Kubo Shigekazu Takemura Takahiro Uenishi Chikaharu Sakata Kazunori Ohata \\ Yorihisa Urata Kazuhisa Kaneda Masayuki Sakae Takayoshi Nishioka Akinori Nozawa \\ Satoshi Kiyota Hiroji Shinkawa
}

\begin{abstract}
Effects of microwave tissue coagulation on postoperative complications in patients who underwent liver resection
\end{abstract}

Shoji Kubo, Shigekazu Takemura, Takahiro Uenishi, Chikaharu Sakata, Kazunori Ohata, Yorihisa Urata, Kazuhisa Kaneda, Masayuki Sakae, Takayoshi Nishioka, Akinori Nozawa, Satoshi Kiyota and Hiroji Shinkawa

\begin{abstract}
In order to evaluate the effects of microwave tissue coagulation (MTC) on postoperative complications, the number of incidences of postoperative bleeding, bile leakage, intraabdominal infection, wound infection, and intractable pleural effusion and ascites were compared between 142 patients who underwent liver resection using MTC (MTC group) and 60 other patients (non-MTC group). The liver was coagulated with a microwave tissue coagulator along the resection line by puncture of the needle applicator (15-mm length) before the transection of the liver. MTC was not used near the thick Glisson's sheath, major hepatic veins, or the hepatic hilum in order to prevent injury to major vessels or bile ducts. There were no differences in the number of incidences of these complications between the groups. In addition, there were no differences in the characteristics of the bacteria isolated between the groups. Thus, MTC does not affect the development of postoperative complications in liver resections when MTC is used appropriately.
\end{abstract}

Key words: liver resection, microwave tissue coagulation, bile leakage, intraabdominal infection, wound infection

Accepted on Aug. 30, 2011

Department of Hepato-Biliary-Pancreatic Surgery, Osaka City University Graduate School of Medicine Address: 1-4-3, Asahimachi, Abeno-ku, Osaka, 545-8585, Japan

Tel: +81-6-6645-3841 Fax: +81-6-6646-6057

Corresponding author: Shoji Kubo, MD

Department of Hepato-Biliary-Pancreatic Surgery, Osaka City University Graduate School of Medicine

E-mail: m7696493@msic.med.osaka-cu.ac.jp 


\section{Introduction}

Since the microwave tissue coagulator was developed and reported by Tabuse et al. ${ }^{1-3)}$, microwave tissue coagulation (MTC) has been applied clinically to the transection of hepatic parenchyma because MTC can reduce blood loss ${ }^{1-5)}$. The usefulness of MTC has also been reported with respect to long-term outcome ${ }^{4) 6}$. However, liver resections with or without MTC can sometimes cause postoperative complications, including postoperative bleeding, bile leakage, and surgical site infection, because of the characteristics of the liver resection itself and the poor liver function of the patients due to chronic liver disease. Ryu et al. ${ }^{4)}$ reported that, of 99 patients who underwent liver resections using MTC, postoperative bleeding developed in 1 patient and bile leakage developed in 9 patients, resulting in a hospital mortality of 3\%. Satoi et al. ${ }^{7}$ reported that, of 214 patients who underwent liver resections using MTC, postoperative bleeding developed in 5 patients, intractable bile leakage developed in 3 patients, and abscess formation on the cut surface of the liver developed in $27 \mathrm{pa}-$ tients, resulting in a hospital mortality of $2.8 \%$.

Recently, the results of liver resections have been improved by advances in diagnostic modalities, surgical techniques, and perioperative management. In this study, the effects of MTC on the occurrence of postoperative complications, such as postoperative bleeding, bile leakage, intraabdominal infection, wound infection, and intractable pleural effusion and ascites, were examined in patients who underwent liver resections in a recent series in our department.

Patients and methods

The subjects in this study were 202 patients who underwent liver resections without reconstruction of the biliary tract and resection of other organs other than the liver between January 2008 and June 2010. Of the 202 patients, 159 had hepatocellular carcinoma, 19 had intrahepatic cholangiocarcinoma, 5 had mixed tumors, 14 had metastatic liver tumors, and 5 had other liver diseases. During surgery, the transection of the liver was performed using an ultrasonic aspirator and bipolar cautery with saline irrigation. Of the 202 patients, the liver was coagulated with a microwave tissue coagulator (Microtaze ${ }^{\circledR}$, Alfresa-pharma Co., Osaka, Japan) along the resection line by the puncture of the needle applicator (15-mm length) before the transection of the liver (precoagulation) in 142 patients. Most of the 142 patients had dysfunction of the liver or had planned to undergo nonanatomic resection. The level of power employed was $80 \mathrm{~W}$, and the coagulation and dissociation times were 30 and $15 \mathrm{sec}$, respectively. MTC was not used near the thick Glisson's sheath, major hepatic veins, or the hepatic hilum in order to prevent injury to major vessels or bile ducts. The clinical findings, operative procedures, and the number of postoperative complications, such as postoperative bleeding, bile leakage, intraabdominal infection, wound infection, intractable pleural effusion and ascites, were compared between the 142 patients in whom MTC was used during surgery (MTC group) and the 60 patients in whom MTC was not used (non-MTC group). Postoperative bile leakage was defined as continuous drainage with a bilirubin concentration above $5 \mathrm{mg} / \mathrm{dL}$ for more than 2 days. Pleural effusion or ascites was defined as intractable when diuretics and thoracocentesis, abdominal paracentesis on 2 or more occasions, or the institution of continuous drainage was necessary for treatment ${ }^{8}$. Infection that occurred during the treatment for bile leakage was not included as an intraabdominal infection.

Results

Although there was no difference in the gender distribution and the prevalence of diabetes mellitus between the groups, age and the prevalence of cirrhosis were significantly higher in the MTC group than in the non-MTC group (Table 1). Although the serum concentrations of total bilirubin and albumin were not different between the groups, the indocyanine green retention rate at $15 \mathrm{~min}$ (ICGR15) and the activities of aspartate aminotransferase (AST) and alanine aminotransferase (ALT) were significantly higher in the MTC group than in the non-MTC group. The platelet count was significantly lower in the MTC group than in the non-MTC group. The number of patients who underwent a nonanatomic resection was significantly higher in the MTC group than in the non-MTC group. The operation time was significantly 
Table 1 Comparison of clinical findings between the groups

\begin{tabular}{|c|c|c|c|}
\hline & $\begin{array}{c}\text { MTC } \\
(n=142)\end{array}$ & $\begin{array}{c}\text { non-MTC } \\
(\mathrm{n}=60)\end{array}$ & $p$ value \\
\hline Gender (male:female) & 104:38 & $44: 16$ & $>0.999$ \\
\hline Age (years, mean $\pm S D$ ) & $69.1 \pm 8.5$ & $66.0 \pm 12.9$ & 0.0493 \\
\hline \multicolumn{4}{|l|}{ Hepatitis virus } \\
\hline Hepatitis B virus & 23 & 15 & \multirow[t]{3}{*}{$<0.0001$} \\
\hline Hepatitis C virus & 82 & 14 & \\
\hline None & 37 & 31 & \\
\hline Diabetes & 29 & 11 & 0.848 \\
\hline Cirrhosis & 47 & 13 & 0.129 \\
\hline Platelet count $\left(\times 10^{4} / \mathrm{mm}^{3}\right)$ & $14.4(7.2,21.5)$ & $16.2(9.4,25.1)$ & 0.0093 \\
\hline Total bilirubin (mg/dL) & $0.8(0.4,1.4)$ & $0.7(0.5,1.2)$ & 0.322 \\
\hline Albumin (g/dL) & $4.0(3.4,4.4)$ & $4.0(3.5,4.5)$ & 0.535 \\
\hline ICGR15 1) (\%) & $15.5(7.5,38.1)$ & $11.3(3.8,26.2)$ & 0.0064 \\
\hline AST (IU/L) & $39(21,72)$ & $31(19,58)$ & 0.0159 \\
\hline $\operatorname{ALT}(I U / L)$ & $31(14,74)$ & $27(12,59)$ & 0.0485 \\
\hline Operation time (min) & $242(129,383)$ & $283(142,474)$ & 0.0285 \\
\hline Blood loss (g) & $475(50,2055)$ & $767(90,2500)$ & 0.0670 \\
\hline Nonanatomical resection & 86 & 14 & $<0.0001$ \\
\hline \multicolumn{4}{|l|}{ Postoperative complication } \\
\hline Bleeding & 0 & 0 & $>0.999$ \\
\hline Bile leakage & 6 & 3 & 0.727 \\
\hline Intraabdominal infection & 6 & 2 & $>0.999$ \\
\hline Wound infection 2) & 4 & 1 & $>0.999$ \\
\hline \multicolumn{4}{|l|}{ Intractable pleural } \\
\hline effusion or ascites & 9 & 5 & $>0.762$ \\
\hline Hospital mortality (\%) & 0 & 0 & $>0.999$ \\
\hline
\end{tabular}

1) Indocyanine green retention rate at $15 \mathrm{~min}$ (ICGR15) was measured in 111 patients in the MTC group and in 47 patients in the non-MTC group.

2) Patients with both wound infection and intraabdominal infection were excluded.

MTC, microwave tissue coagulation; AST, aspartate aminotransferase; ALT, alanine aminotransferase.

shorter in the MTC group than in the non-MTC group. The amount of intraoperative blood loss was significantly smaller in the MTC group than in the non-MTC group. As described in the "Patients and methods" section, MTC was mainly performed in patients who had dysfunction of the liver or who had planned to undergo nonanatomic resection in order to reduce blood loss during transection of the liver.

Postoperative bleeding did not occur in either group. Bile leakage occurred in 6 patients in the MTC group and in 3 patients in the non-MTC group. The number of incidences of bile leakage was not different between the groups. These 9 patients had at least 1 factor that has been reported to contribute to bile leakage, such as a large tumor near the hepatic hilum, resection of the caudate lobe, nonanatomic resection, or repeated liver resection ${ }^{9)}$. Thus, MTC did not directly affect the development of bile leakage. Intraabdominal infection developed in 6 patients in the MTC group and in 2 patients in the non-MTC group. Wound infection developed in 4 patients in the MTC group and in 1 patient in the non-MTC group. The number of incidences of intraabdominal infection and wound infection were not different between the groups. In the MTC group, Methicillin-resistant Staphylococcus aureus (MRSA), Methicillin-sensitive S. aureus, Methicillin-resistant coagulase-negative Staphylococcus spp. (MRCNS), Enterococcus faecalis, or E. gallinarum was isolated from the drainage fluid from the intraabdominal abscess. In the non-MTC group, $E$. faecalis, Klebsiella pneumoniae, or Pseudomonas aeruginosa was isolated. In the MTC group, MRSA, S. epidermidis (MRCNS), Escherichia coli, or Corynebacterium was isolated from the infectious wound. In the non-MTC group, Corynebacterium was isolated from the infectious wound. One of the 2 patients in whom MRSA was isolated was thought to have MRSA before surgery because MRSA was detected in the various samples obtained immediately after surgery. Thus, the isolated bacteria mainly originated from the intestinal or skin flora in both groups, and there was no difference in the characteristics of the bacteria isolated between the groups. There was no difference in the number of incidences of intractable pleural effusion and ascites between the groups. Hospital mortality was $0 \%$ in both groups.

\section{Discussion}

In our department, MTC has been used to reduce blood loss during transections of the liver, mainly in nonanatomic resections in patients with chronic liver disease, including liver cirrhosis. Although the usefulness of MTC has been reported by several investigators ${ }^{1)-6)}{ }^{9)}$, MTC may cause postoperative bleeding, bile leakage from injury of the bile ducts, and intraabdominal abscesses because MTC produces coagulonecrotic tissue along the cut surface of the liver. In fact, these complications have been reported in previous studies ${ }^{4) 6}$.

In this study, although the backgrounds of the patients 
were different between the MTC group and the non-MTC group, the number of incidences of bile leakage, intraabdominal infection, wound infection, intractable pleural effusion or ascites was not different between the groups. Postoperative bleeding did not occur in either group. Thus, MTC does not strongly affect the development of such complications. In our department, MTC was not used near the thick Glisson's sheath, major hepatic veins, or near the hepatic hilum in order to prevent injury to major vessels or bile ducts. It is important to pay attention to this in order to prevent serious complications by MTC.

MTC has been used for ablation therapy in percutaneous procedures ${ }^{3)}$, , open surgery ${ }^{3)}{ }^{11-13)}$, and laparoscopic sur-

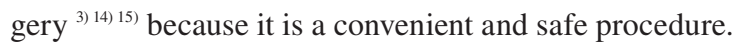

In conclusion, MTC did not affect the development of postoperative complications in liver resections when MTC was used appropriately.

\section{References}

1) Tabuse K: A new operative procedure of hepatic surgery using a microwave tissue coagulator. Nihon Geka Hokan 48: 160-172, 1979

2) Tabuse K, Katsumi M, Kobayashi Y, Noguchi H, Egawa H, Aoyama O, Kim H, Nagai Y, Yamaue H, Mori K, Azuma Y, Tsuji T: Microwave surgery: hepatectomy using microwave tissue coagulator. World J Surg 9: 136-143, 1985

3) Tabuse K: Basic knowledge of a microwave tissue coagulator and its clinical applications. J Hepatobiliary Pancreat Surg 5: 165-172, 1998

4) Ryu M, Watanabe K, Yamamoto H: Hepatectomy with microwave tissue coagulation for hepatocellular carcinoma. J Hepatobiliary Pancreat Surg 5: 184-191, 1998

5) Reuter NP, Martin II RCG: Microwave energy as a precoagulative device to assist in hepatic resection. Ann Surg Oncol 16: 3057-3063, 2009

6) Satoi S, Matsui Y, Kitade H, Yanagimoto H, Toyokawa H, Yamamoto H, Hirooka S, Kwon AH, Kamiyama Y: Long-term outcome of hepatocellular carcinoma patients who underwent liver resection using microwave tissue coagulation. HPB (Oxford) 10: 289-295, 2008

7) Satoi S, Kamiyama Y, Matsui Y, Kitade H, Kaibori M, Yamamoto H, Yanagimoto H, Takai S, Kwon AH: Clinical outcome of 214 liver resections using microwave tissue coagulation. Hepatogastroenterology 52: 1180-1185, 2005

8) Kubo S, Tsukamoto T, Hirohashi K, Tanaka H, Shuto T, Takemura S, Yamamoto T, Uenishi T, Ogawa M, Kinoshita H: Correlation between preoperative serum concentration of type IV collagen 7s domain and hepatic failure following resection of hepatocellular carcinoma. Ann Surg 239: 186-193, 2004

9) Kubo S, Takemura S, Uenishi T, Kiyota S, Shinkawa H, Urata Y, Ohata K, Kaneda K, Sakae M, Nozawa A, Kodai S, Kanazawa A, Tsukamoto T: Abdominal drainage after liver resection. Jpn J Portal Hypertension 17: 52-55, 2011

10) Katsuragi K, Hirohashi K, Sakata C, Kubo S, Tanaka H, Tsukamoto T, Shuto T, Kinoshita H: Day surgery percutaneous microwave coagulation therapy for hepatocellular carcinoma. J Microwave Surg 17: 49-53, 1999

11) Yamanaka N, Tanaka T, Oriyama T, Furukawa K, Tanaka W, Okamoto E: Microwave coagulonecrotic therapy for hepatocellular carcinoma. World J Surg 20: 1076-1081, 1996

12) Tanaka H, Kinoshita H, Hirohashi K, Kubo S, Tsukamoto T, Shuto T, Ikeda K, Takemura S, Botani T, Kiyota S, Okuda T, Kanazawa A, Mikami S: Microwave coagulo-necrotic therapy for unresectable hepatocellular carcinoma. J Microwave Surg 13: 83-87, 1995

13) Yamamoto T, Sakabe K, Tanaka S, Uenishi T, Ogawa M, Ichikawa T, Hai S, Ito T, Kato H, Ishihara K, Kubo S, Ito S, Hirohashi K: Microwave coagulation therapy with surgical technique for hepatocellular carcinoma. J Microwave Surg 22: 79-84, 2004

14) Sakaguchi H, Morikawa H, Tamori A, Habu D, Nishiguchi S, Shiomi S, Seki S, Kuroki T, Hanba H, Kubo S, Hirohashi K, Kinoshita H: Treatment of hepatocellular carcinoma with use of microwave coagulation under laparoscopy. J Microwave Surg 17: 89-93, 1999

15) Tsuchiya M, Otsuka Y, Tamura A, Nitta H, Sasaki A, Wakabayashi G, Kaneko H: Status of endoscopic liver surgery in Japan: a questionnaire survey conducted by the Japanese Endoscopic Liver Surgery Study Group. J Hepatobiliary Pancreat Surg 16: 405-409, 2009 